\title{
Approximation of a Mathematical Aging Function for Latent Fingerprint Traces Based on First Experiments Using a Chromatic White Light (CWL) Sensor and the Binary Pixel Aging Feature
}

\author{
Ronny Merkel ${ }^{1}$, Jana Dittmann ${ }^{1}$, and Claus Vielhauer ${ }^{2}$ \\ ${ }^{1}$ Department of Computer Science, Research Group Multimedia and Security, \\ Otto-von-Guericke-University of Magdeburg, Universitätsplatz 2, 39106 Magdeburg, Germany \\ ${ }^{2}$ Department of Informatics and Media, Brandenburg University of Applied Sciences, \\ Magdeburger Straße 50, 14770 Brandenburg an der Havel, Germany \\ \{Ronny.Merkel, Jana.Dittmann\} @iti.cs.uni-magdeburg.de, \\ Claus.Vielhauer@fh-brandenburg.de
}

\begin{abstract}
The age determination of latent fingerprint traces is a very important challenge for forensic investigations, which has not been solved satisfyingly so far. Based on prior work, we use the novel and very promising aging feature of counting binary pixel for the approximation of a mathematical aging function to be used for the age determination of latent fingerprint traces. We first show the feasibility of this feature in a test set of nine test series (each comprised of a fingerprint sample scanned continuously over four days) using three different optical sensors (CWL) of the same model and varying resolutions $(3,5,10 \mu \mathrm{m})$. We then approximate the aging function for each test series, showing an average error of approximation between $13 \%$ and $40 \%$ for an optimal approximation. We discuss the prospects and restrictions of such a function for the age determination of latent fingerprint traces and identify future research challenges.
\end{abstract}

Keywords: latent fingerprint traces, crime scene investigation, age determination, binary pixel, mathematical aging function.

\section{Introduction}

The determination of the age of a latent fingerprint trace found at a crime scene is a strong need in forensic investigations since many decades. Only very limited results have been achieved in this field so far. In recent years, contactless scanning techniques have been introduced to the field of fingerprint forensic research, often adapting surface- or other purpose measurement devices for the non-invasive acquisition of latent fingerprint traces (see [1] for a summary of such techniques). These acquisition techniques offer new opportunities to the field of fingerprint age determination, since latent fingerprint traces can now be obtained with a very high resolution and accuracy (which could not fully be achieved by classical powdering and sticky tape lifting or fuming with cyanoacrylate or other development techniques and subsequent photographing). 
In [2], we suggested the measurement of 'binary pixel' as a first feature for a possible age determination, using a contact-less, non-invasive Chromatic White Light (CWL) sensor [3] as part of a surface measurement device for acquiring latent fingerprint traces from a hard disc platter. To the best of our knowledge, this is a novel approach, firstly using high-resolution image sensory in combination with pattern recognition techniques, which seems to be very promising to finally solve the important research challenge of the age determination of latent fingerprint traces. The technique normalises the captured fingerprint image, binarises it using a threshold and then counts the white pixel, which represent the background pixel not belonging to the residue. We show that the amount of this white pixel increases in a logarithmic way over time. This increase is assumed to happen due to decomposition processes of the fingerprint residue and the evaporation of water. In our design approach from [2] we scan a single fingerprint sample continuously over a time period of 10 hours, referred to as a test series throughout this paper. We examine only four of such test series in [2] using one CWL device (for each series, an individual left a fingerprint trace on a hard disc platter) and give no specific details on the parameters of the observed aging curve. Here, we extend these considerations by proposing a mathematical aging function which might be used for the age determination of latent fingerprint traces and evaluate the reproducibility of the characteristic tendency of the aging feature using different resolutions and different sensors of the same model as well as an increased test series length of four days.

Our first test goal is to reproduce the results of [2] using an extended test set of nine test series, where each test series is comprised of a fingerprint left on a hard disc platter by an individual (randomly chosen from four different individuals providing their fingerprints for the tests) which is continuously scanned over four days (with a scan interval of 30 minutes or less, depending on the resolution). For this nine test series, we vary the resolution $(3 \mu \mathrm{m}, 5 \mu \mathrm{m}$ and $10 \mu \mathrm{m})$ and use three different CWL sensors of the same model (FRT MicroProf 200 CWL 600 [3], which is identical to the one used in [2]) to evaluate the reproducibility of the characteristic tendency of the feature under different scanning conditions.

Our second test goal is to then approximate a mathematical aging function from our test results, which might later be used for the determination of the absolute age of a fingerprint trace. Based on the standard mathematical representation of a natural logarithmic curve $f(t)=a * \ln (t)+b, t>0$ we approximate the parameters $a$ and $b$ for each test series. We then evaluate the average error of approximation of such an approximated mathematical function, showing no significant tendency for the influence of different scanners of the same model or different resolutions within the test range, but significant variations between the results of the test series (average errors of approximation between $13 \%$ and $40 \%$ ), possibly caused by different influences such as the sweat composition of the residue (e.g. type and duration of sweating before fingerprint application or consumption of medicine, certain food or other substances), the environmental conditions (e.g. UV-light, temperature or humidity) or fingerprint application related influences (such as smearing or blurring of the fingerprint or contact time and -pressure when applying the print).

We discuss the potential use of our approximated aging function in a forensic application scenario and identify future research challenges, which need to be solved for achieving a certain accuracy of the results. 
The paper is structured as follows: in the next section, we give a short summary of current state of the art approaches concerning the topic and present our used sensor. We then introduce our design approach for a mathematical aging function in section 3 , which might be used for the age determination of latent fingerprint traces. In section 4, we explain our assumptions, present our test setup and evaluate the test results for our two test goals. We afterwards discuss the potential application of an approximated mathematical aging function in a forensic investigation in section 6 . Section 7 summarises the paper and identifies future work.

\section{State of the Art}

So far, only very limited progress has been made in the domain of age determination of latent fingerprints in forensics. A few approaches include the use of fingerprint ridge thickness and changes of pores [4], the healing stage of a wound on the finger [5], fluorescence-properties of a fingerprint changing over time [6] or the use of a reference database of fingerprints of different aging states [7]. A good summary of current approaches for the age determination of latent fingerprints can be found in the German study of [6]. However, all of these approaches are either focused on very limited scenarios (e.g. a wound on the finger is required) or fail to deliver reliable results. They can therefore not satisfy the strong need for a comprehensive age determination of latent fingerprint traces.

In prior work [2], we reported that a novel feature called 'binary pixel' shows a very promising, characteristic logarithmic aging curve for latent fingerprint traces left on a hard disc platter. The experiments are conducted using the FRT MicroProf 200 CWL 600 sensor [3] for acquiring the prints and four different fingerprint samples are captured continuously over 10 hours. For determining the binary pixel value, we normalise a scanned fingerprint image and binarise it using a threshold. We then measure the number of white background pixel in relation to the number of overall pixel, which results in our feature value for the binary pixel feature.

Since the feature of counting binary pixel seems to be very characteristic for the aging process of latent fingerprint traces, we want to use it for our idea of approximating a mathematical aging function, which might be used for the age determination of latent fingerprint traces.

To reproduce the characteristic tendency in our extended test set, we use the same FRT MicroProf 200 CWL 600 measurement device, which origins from the field of quality control of materials and is transferred to the domain of fingerprint imaging. It uses a chromatic white light (CWL) sensor and can produce high-resolution 16-bit intensity- and topography images of a fingerprint trace with a resolution of up to $1 \mu \mathrm{m}$ in the lateral domain and $20 \mathrm{~nm}$ in the longitudinal domain. For our research, so far only the intensity images produced by the sensor are of interest, leaving the use of topographical values for future work. Potential other contactless sensors are summarised in [1].

\section{Our Proposed Design for an Aging Function}

For the approximation of a mathematical aging function for a given aging feature, the general tendency of the course of the features aging curve needs to be known. To the 
best of our knowledge, most natural processes have a logarithmic, exponential or (in some cases) linear course. We identified the course of the aging curve for the binary pixel feature as logarithmic. This tendency was confirmed by all nine test series examined in this paper. We therefore approximate the course of the aging curve by using the standard mathematical formula for a logarithmic function:

$$
f(t)=a * \log _{c}(t)+b, t>0 .
$$

The variables of the function (1) are represented by a point in time $t$ and the relative amount of white background pixel $f(t)$ present at this point. The constant parameters $a, b$ and $c$ describe specific aspects of the function and need to be determined for a specific aging curve. The parameter $c$ describes the base of the logarithm and, since the aging of fingerprint traces is a natural process, is assumed to be Euler's number (e), leading to formula (2). However, the function could be approximated using other values for the base $c$ in an analogous way.

$$
f(t)=a * \ln (t)+b, t>0 .
$$

Although the mathematical aging function for the given aging feature is to be approximated, it is trivially not identical to the experimentally determined aging curve described by the test results. Therefore, we have to distinguish between a tuple $t u$ $p_{E i}=\left(t_{E i}, f_{E}\left(t_{E i}\right)\right), t_{E i}>0$ which describes a specific point in time $t_{E i}$ and its corresponding amount of white background pixel $f_{E}\left(t_{E i}\right)$ from the discrete experimental aging curve $f_{E}$ (where $i$ describes the index number of the tuple within a test series) and the tuple $\operatorname{tup}_{M i}=\left(t_{M i} f_{M}\left(t_{M i}\right)\right), t_{M i}>0$, which represents the corresponding point in time $t_{M i}$ and its value $f_{M}\left(t_{M i}\right)$ as part of the continuous mathematical function $f_{M}$ approximating the course of the aging curve. Considering this naming scheme, formula (2) can be rewritten as:

$$
f_{M}\left(t_{M i}\right)=a * \ln \left(t_{M i}\right)+b, t_{M i}>0 .
$$

Furthermore, the experimental results of a test series described by the discrete aging function $f_{E}$ consist of a set of tuples $T_{E}=\left\{\left(t_{E 1}, f_{E}\left(t_{E 1}\right)\right),\left(t_{E 2}, f_{E}\left(t_{E 2}\right)\right), \ldots,\left(t_{E n}, f_{E}\left(t_{E n}\right)\right) ; t_{E i}>0\right.$, $n \in N, n>0\}$ representing all $n$ measured samples of a fingerprint trace.

Formula (3) can be transposed to calculate the point in time $t_{M i}$ of a sample from the amount of white background pixel $f_{M}\left(t_{M i}\right)$ present:

$$
t_{M i}=e^{\frac{f_{M}\left(t_{M i}\right)-b}{a}}, a \neq 0 .
$$

By substituting $f_{M}\left(t_{M i}\right)$ with its experimental equivalent $f_{E}\left(t_{E i}\right)$, we derive the following formula:

$$
t_{M i}{ }^{\prime}=e^{\frac{f_{E}\left(t_{E i}\right)-b}{a}}, a \neq 0 .
$$

The derived formula (5) enables us to calculate the absolute amount of time $t_{M i}$ ' which has passed from the time a fingerprint trace was left on a surface until the acquisition of a binary pixel feature value $f_{E}\left(t_{E i}\right)$, given that the constant parameters $a$ and $b$ are 
known. However, since influences like environmental conditions (which are discussed in section 4.1) might change over time and also other influences (such as sensor noise) are present, there is an offset between the calculated theoretical age $t_{M i}$ ' of a fingerprint trace and its real age $t_{E i}$. This offset characterises the error of approximation $\operatorname{err}_{i}$ for a specific tuple $\operatorname{tup}_{E i}$ :

$$
\operatorname{err}_{i}=\frac{\left|t_{E i}-t_{M i}^{\prime}\right|}{t_{E i}}, t_{E i}>0 .
$$

To obtain an average error of approximation err $_{\text {avg }}$ for a complete test series, we determine the errors $e r r_{i}$ for all tuples $t u p_{E i}$ and calculate their average value:

$$
\begin{gathered}
\operatorname{err}_{\text {avg }}=\frac{1}{n} * \sum_{i=1}^{n} \operatorname{err}_{i}=\frac{1}{n} * \sum_{i=1}^{n} \frac{\left|t_{E i}-t_{M i}{ }^{\prime}\right|}{t_{E i}}=\frac{1}{n} * \sum_{i=1}^{n} \frac{\mid t_{E i}-e^{\frac{f_{E}\left(t_{E i}\right)-b}{a} \mid}}{t_{E i}} . \\
n \in N, n>0, t_{E i}>0, a \neq 0
\end{gathered}
$$

By using this formula, the average error of approximation err $_{\text {avg }}$ for an arbitrary test series can be calculated. In the following section, we evaluate the accuracy of such an approximation by determining the parameters $a$ and $b$ in a way to minimise the average error $e r r_{\text {avg }}$.

\section{Experimental Investigations}

Changing influences on the fingerprint aging process are supposed to be the biggest source of impreciseness of our approximated aging curve. Furthermore, some influence factors might have a big impact resulting from only small changes whereas other influence factors are rather insignificant. In this section, we therefore first introduce our main assumption concerning these influences, which is according to our knowledge a precondition for a reliable age determination. We then introduce our experimental test setup and evaluate the measured results regarding our two test goals: the reproduction of the characteristic aging tendency using an extended test set and the approximation of a mathematical aging function from our test results.

\subsection{Assumptions}

Many different influences exist on the aging process of a latent fingerprint trace (see [4], [5], [6], [7], [8], [9], [10], [11] and [12]). We summarise them here as: the sweat influence (namely all factors influencing the consistency of the sweat and therefore its decomposition process), the environmental influence (such as UVlight, humidity, temperature, wind or air pressure), the application influence (which is determined by the characteristic way a fingerprint trace is left, such as smearing or blurring, contact-time, contact pressure or the application of creme or 
dirt to a finger prior to applying the fingerprint) and the surface influence (where characteristic surface properties influence the aging process, such as absorptive or corroding surfaces). Furthermore, the scan influence of the fingerprint acquisition process, meaning the influence of the technical properties of the capturing device and the acquisition parameters (such as the resolution of a scan or the size of the measured area), can have an important influence on the determination of the aging function of a given feature, especially if chosen wrong.

In this paper, we assume that the sweat influence, the environmental influence and the application influence are kept constant. We are not interested in the explicit values of these influences (e.g. if the temperature is $20^{\circ} \mathrm{C}$ or $30^{\circ} \mathrm{C}$ ) as long as they are constant. However, it might not be totally possible in practice to keep all this influences constant, since they are subject to changes over time, such as temperature and humidity changes or differences in the sweat composition of different fingerprint traces. Therefore, the resulting error of approximation of the aging function calculated in the next sections might be partially a result of such influences not being totally constant. It might be improved in future work by taking additional arrangements to keep all influences, which are not subject of the study, as constant as possible, or taking such influences into account when determining the age of a fingerprint.

Other influences, such as the surface influence, can be kept constant very well by fixing the used material to a hard disc platter used for all tests.

The scan influence of the fingerprint acquisition process is subject to investigation in the scope of our first test goal (see section 1) and is therefore systematically varied in the next section to experimentally determine the significance of its influence.

\subsection{Test Setup}

For our test setup we designed nine different test series (see table 1). Each test series is comprised of one fingerprint sample from an individual (randomly chosen from four individuals contributing their fingerprints to the experiments) left on a hard disc platter (constant surface influence, ideal surface for the used measurement device), of which a randomly selected part of the size $2.5 \times 2.5 \mathrm{~mm}$ is continuously scanned over a total period of four days (with a scan interval of 30 minutes or less, depending on the resolution). The size of the measured area and the scan interval are chosen in respect to the performance of the CWL sensor. At a resolution of $3 \mu \mathrm{m}$, a size of the measured area of $2.5 \times 2.5 \mathrm{~mm}$ can be scanned in 30 minutes. If the scan interval is to be increased, the size of the measured area or the resolution needs to be decreased and vice versa. A size of the measured area of $2.5 \times 2.5 \mathrm{~mm}$ furthermore seems to be very sufficient for calculating the binary pixel feature (containing approximately between 5 and 8 ridge lines) and might even be decreased in future work. It is furthermore assumed that a total scan period of four days is sufficient for a first approximation of the aging function, since most changes to the fingerprint are happening within the first hours (which is already mentioned in [2] and is confirmed in our test results). For a more precise approximation, longer scanning periods might also be of interest in future work. Figure 1 exemplary depicts five different scan images of our test series 1 , captured at different points in time $t_{1}=0$ days, $t_{2}=1$ day, $t_{3}=2$ days, $t_{4}=3$ days and $\mathrm{t}_{5}=4$ days . 


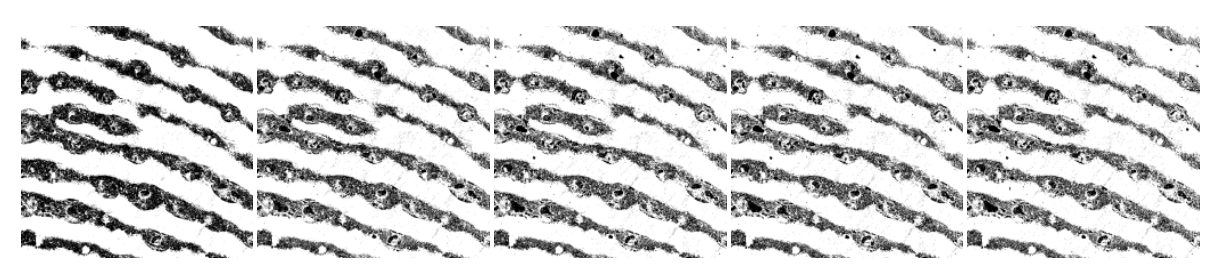

Fig. 1. Captured fingerprint images from test series 1 at five different points in time (from left to right): $\mathrm{t}_{1}=0$ days, $\mathrm{t}_{2}=1$ day, $\mathrm{t}_{3}=2$ days, $\mathrm{t}_{4}=3$ days and $\mathrm{t}_{5}=4$ days

The used CWL sensor as well as the resolution of scans captured within a test series is varied to extract possible influences of these factors (scan influence). While the intensity images of three different CWL sensors of the same model (FRT MicroProf 200 CWL 600; see [3]) are used, the resolution of the test series for each of these sensors is varied between 3,5 and $10 \mu \mathrm{m}$.

For all test series, the 16bit grey scale intensity images acquired by the sensor are normalised to the interval $[0 ; 1]$ and are binarised using the threshold thresh $=0.8$. Since we use only similar hard disc platter in our experiments, such general threshold is applicable to all test series, which has been confirmed in preliminary tests. However, for using different types of platter or different surfaces in future work, the threshold might have to be adapted to different pixel grey value distributions.

Table 1. Overview of our experimental test setup for nine test series, each comprised of one fingerprint trace on a hard disc platter, of which a randomly selected area of $2.5 \times 2.5 \mathrm{~mm}$ is measured in intervals of 30 minutes or less (depending on the resolution) over four days

\begin{tabular}{|c|c|c|c|c|c|c|c|c|c|c|}
\hline \multicolumn{2}{|c|}{ Test series number } & $\mathbf{1}$ & $\mathbf{2}$ & $\mathbf{3}$ & $\mathbf{4}$ & $\mathbf{5}$ & $\mathbf{6}$ & $\mathbf{7}$ & $\mathbf{8}$ & $\mathbf{9}$ \\
\hline \multirow{2}{*}{$\begin{array}{c}\text { Scan } \\
\text { influence }\end{array}$} & Scanning & CWL & CWL & CWL & CWL & CWL & CWL & CWL \\
device & 1 & 1 & 1 & 2 & 2 & 2 & 3 & 3 & CWL \\
\cline { 2 - 11 } & resolution & $3 \mu \mathrm{m}$ & $5 \mu \mathrm{m}$ & $10 \mu \mathrm{m}$ & $3 \mu \mathrm{m}$ & $5 \mu \mathrm{m}$ & $10 \mu \mathrm{m}$ & $3 \mu \mathrm{m}$ & $5 \mu \mathrm{m}$ & $10 \mu \mathrm{m}$ \\
\hline
\end{tabular}

Given the introduced scan interval ( $\leq 30 \mathrm{~min})$ and scan period (4 days), a total of at least 192 tuples $\left(t_{E i}, f_{E}\left(t_{E i}\right)\right)$ of different times $t_{E i}$ and corresponding relative amounts of white background pixel $f_{E}\left(t_{E i}\right)$ can be extracted for each test series, which can be used for the approximation of the mathematical aging function and the corresponding error of approximation described in section 3.

\subsection{Experimental Results}

The reproduction of the logarithmic course of a fingerprint aging curve using the binary pixel feature was introduced as our first test goal in section 1 . For all nine test series, a clear logarithmic course of the aging curve can be seen in figure 2 . Therefore, the basic assumption for the approximation of our aging function is shown to be valid. We also re-sketched the experimental aging-curve from the investigations of [2] for comparison (see figure 2). 


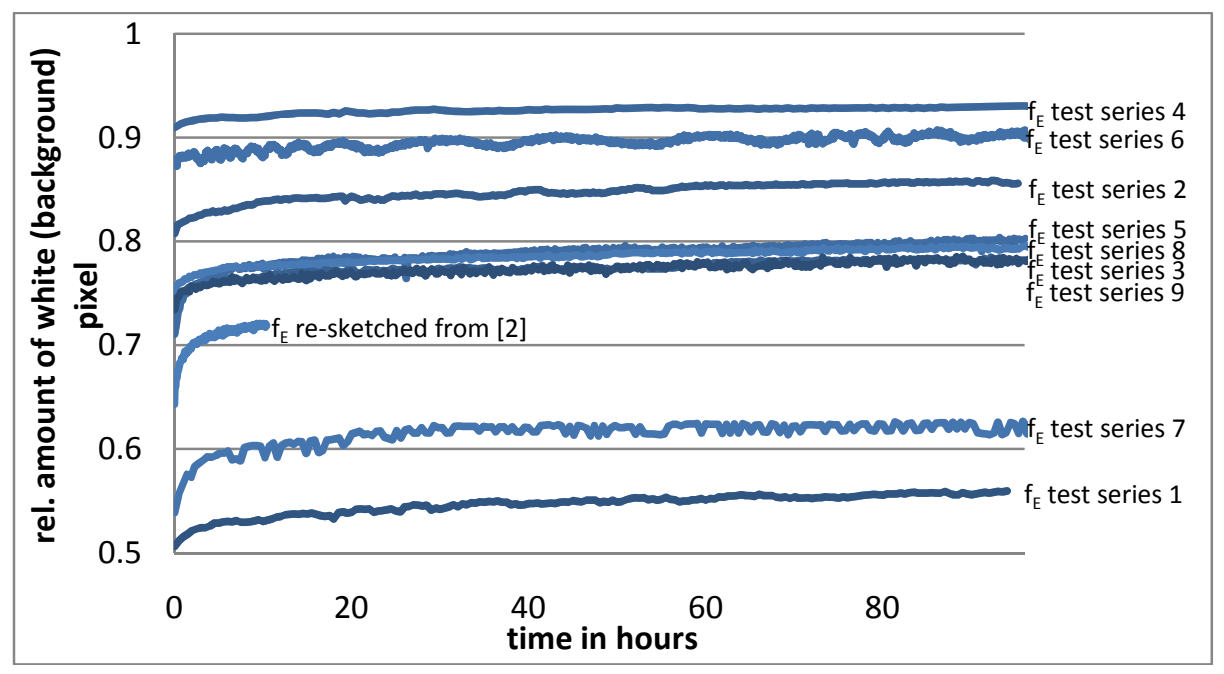

Fig. 2. The experimental aging curves for our nine test series depicting the relative amount of white background pixel in relation to the time passed; the experimental results from [2] are also re-sketched for comparison

The test results show that all experimental aging curves for the binary pixel feature have a logarithmic course and that the biggest changes happen within the first hours. They therefore confirm the results presented in [2] and furthermore show that there are differences in the slopes of the curves. Also, the curves are shifted along the $y$-axis. This is most likely caused by the different amounts of residue present for the different test series, since different latent fingerprint traces bear different amounts of residue (due to sweat composition, contact pressure when leaving a print, or other influences).

Comparing the experimental aging curves of different resolutions and different CWL sensors frome these first experiments, no clear dependency can be found on either the resolution or the used sensor. However, the distortion of some curves (such as the ones from test series 6 and 7 depicted in fig. 2) is much higher than that of others, which seems to be a result of environmental influences (such as temperature, humidity or wind) which can not totally be controlled during our current experiments but should be taken into account in future research.

In our second test goal from section 1 we want to approximate the parameters $a$ and $b$ of our formula (7) to calculate and minimise the average error of approximation $\operatorname{err}_{\text {avg }}$ (see also section 3). For each test series, we have at least 192 tuples $\operatorname{tup}_{E i}$ (as calculated in section 4.2), each consisting of a point in time $t_{E i}$ and the corresponding relative amount of white background pixel $f_{E}\left(t_{E i}\right)$.

The approximation of $a$ and $b$ can be seen as an optimisation problem. More precise, we want to find the minimum value for the average error err $_{\text {avg }}$ described by function (7) for varying parameters $a$ and $b$. We solve this minimisation problem with the help of a computer program, which is systematically varying $a$ and $b$ in steps of $1 / 10000$ in the interval of $[0,1]$ and determines the values of $a$ and $b$ for which err $_{\text {avg }}$ is minimal. With the optimised parameters $a$ and $b$ we can approximate the course of 
the discrete experimental aging curve by our derived continuous mathematical curve (see figure 3). The optimised parameters $a$ and $b$ as well as their corresponding average error $e r r_{\text {avg }}$ are listed in table 2 for all test series.

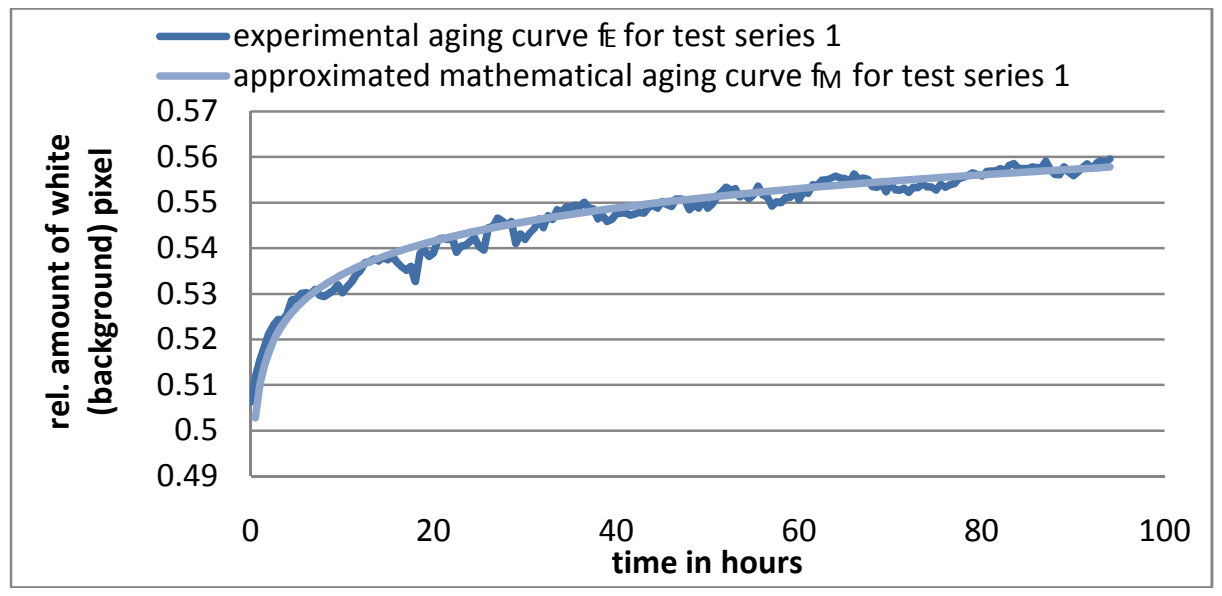

Fig. 3. Exemplary illustration of the experimental aging curve and the approximated mathematical aging curve for the aging feature of counting binary pixel, shown for test series 1 . The relative amount of white background pixel is shown in relation to the time passed.

In figure 3 we illustrate for test series 1 that the derived mathematical aging function seems to approximate the average course of the experimental curve very well, leaving offsets (which are probably due to the influence factors described in section 4.1 not being totally constant) of the practically measured white background pixel to both sides of the curve.

Table 2. Our experimental test results for the optimized values of the parameters $a$ and $b$ as well as the average error of approximation $\operatorname{err}_{\text {avg }}$ for all test series

\begin{tabular}{|c|c|c|c|c|c|c|c|c|c|}
\hline $\begin{array}{c}\text { Test series } \\
\text { number }\end{array}$ & $\mathbf{1}$ & $\mathbf{2}$ & $\mathbf{3}$ & $\mathbf{4}$ & $\mathbf{5}$ & $\mathbf{6}$ & $\mathbf{7}$ & $\mathbf{8}$ & $\mathbf{9}$ \\
\hline $\begin{array}{c}\text { Scanning } \\
\text { device }\end{array}$ & CWL1 & CWL1 & CWL1 & CWL2 & CWL2 & CWL2 & CWL3 & CWL3 & CWL3 \\
\hline resolution & $3 \mu \mathrm{m}$ & $5 \mu \mathrm{m}$ & $10 \mu \mathrm{m}$ & $3 \mu \mathrm{m}$ & $5 \mu \mathrm{m}$ & $10 \mu \mathrm{m}$ & $3 \mu \mathrm{m}$ & $5 \mu \mathrm{m}$ & $10 \mu \mathrm{m}$ \\
\hline Optimal a & 0.0105 & 0.0093 & 0.0071 & 0.0036 & 0.0112 & 0.0059 & 0.0119 & 0.0079 & 0.0080 \\
\hline Optimal b & 0.5101 & 0.8151 & 0.7504 & 0.9133 & 0.7489 & 0.8764 & 0.5752 & 0.7573 & 0.7454 \\
\hline err $_{\text {avg }}$ & 0.1437 & 0.1548 & 0.2006 & 0.1689 & 0.1319 & 0.3988 & 0.2991 & 0.1652 & 0.2580 \\
\hline
\end{tabular}

The test results in table 2 show three findings:

a) No clear dependency on either the resolution of a test series or the used sensor within the test range. For neither the parameters $a$ or $b$, nor the average error of approximation err $_{\text {avg }}$ any clear tendency can be extracted, which might be related to the resolution or the used sensor. Therefore, we can assume that the differences in the test results are caused by influences other than the scan influence. 
b) The values of the parameters $a$ and $b$ vary greatly. While the parameter $b$ is dependent on the relative amount of residue in relation to the overall pixel (as mentioned earlier) and therefore is expected to vary in dependence of the fingerprint trace, the high variation in the values of the parameter $a$ is very challenging to explain. Here, as mentioned already in section 4.1 , changing influences such as the environmental influence (e.g. temperature, humidity or wind) or the sweat influence (e.g. the composition of the residue) might have a strong impact.

c) The average error of approximation $e r r_{a v g}$ takes on values between $13-40 \%$ for all test series. This shows that under the given conditions, even if the optimal values for the parameters $a$ and $b$ can be determined, this error is the best we can get. The reason for this error might be fluctuations in the amount of white background pixel $f_{E}\left(t_{E i}\right)$ measured for a certain point in time $t_{E i}$, which can be a result of changing influences (such as temperature changes, humidity changes or wind) where slight changes might be enough to change the decomposition speed of the fingerprint sample or even affect the sensors capturing characteristics. Therefore, the error might be improved in future work by taking additional measures to keep all influences more constant or to take their changes into account. Of course, if the age of a fingerprint trace is to be determined at a crime scene, these changes of influences might not always be controllable or reproducible.

Furthermore, examining the error of approximation $e r r_{i}$ between experimental points in time $t_{E i}$ and their calculated theoretical equivalents $t_{M i}$ shows varying results. Figures 4 and 5 exemplary represent two characteristic tendencies, which occurred for the test series. In some cases (see figure 4), the error of approximation $\operatorname{err}_{i}$ is very high in the beginning and decreases over time, whereas in other cases (see figure 5) it is very unpredictable. This confirms the assumption that the error might be a result of fluctuations in the external influences, since they are expected to occur randomly.

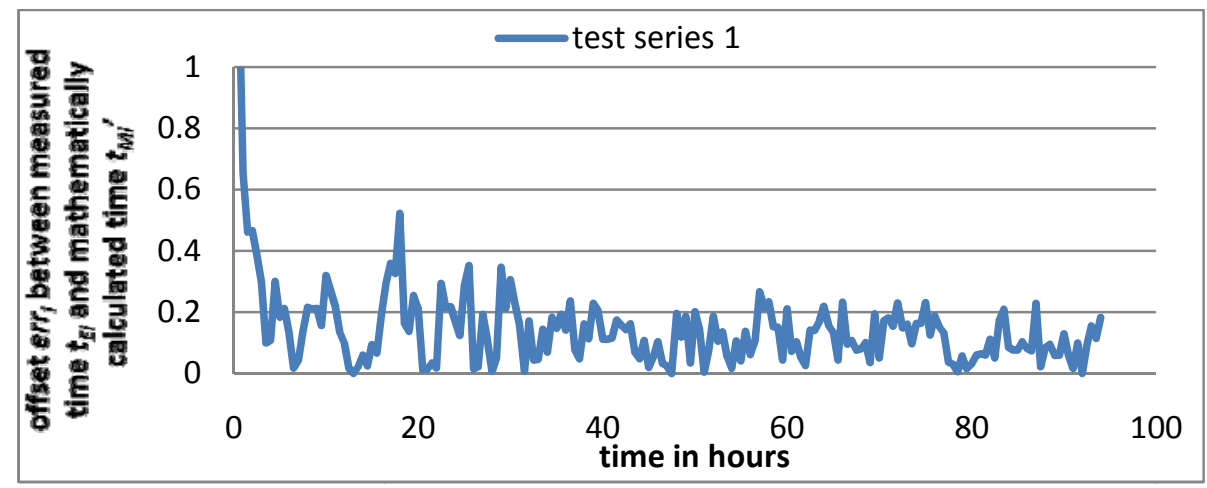

Fig. 4. Our experimental results for the error of approximation $\operatorname{err}_{i}$ between the measured time $t_{E i}$ and the mathematically calculated time $t_{M i}$ ' of a sample in relation to the absolute time passed, exemplary shown for test series 1 


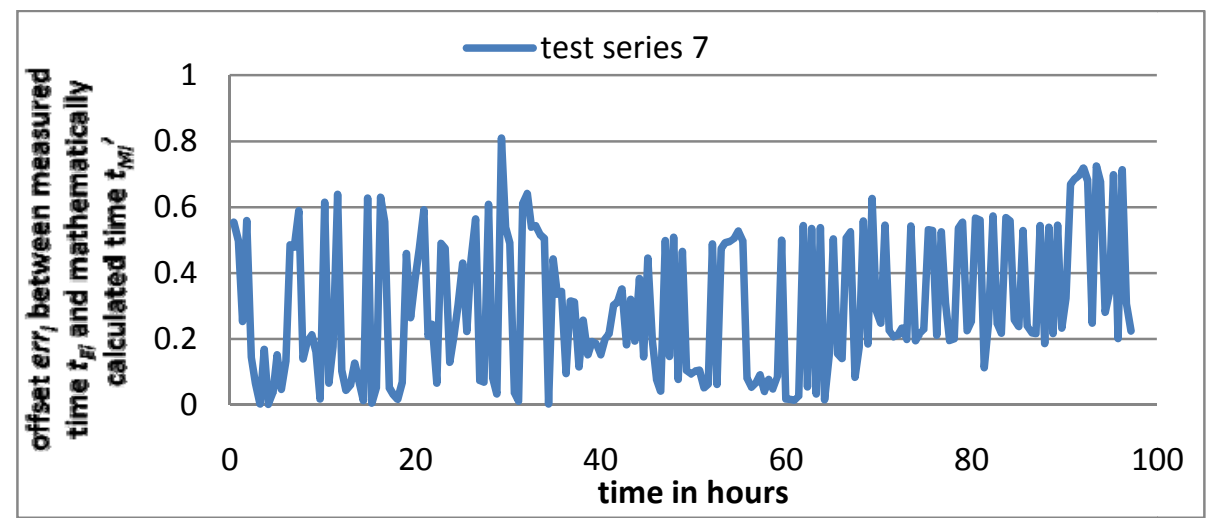

Fig. 5. Our experimental results for the error of approximation $\operatorname{err}_{i}$ between the measured time $t_{E i}$ and the mathematically calculated time $t_{M i}$ ' of a sample in relation to the absolute time passed, exemplary shown for test series 7 .

The other test series show similar tendencies in the progression of their error of approximation if compared to the exemplary depicted ones. Both tendencies occur nearly equally often amongst the test series.

\section{Discussion}

For our mathematical aging function to be usable in a forensic application scenario (e.g. at a crime scene), the constant parameters $a$ and $b$ play a major role. If they can be derived correctly, we can determine the age of a fingerprint with an average error of approximation err $_{\text {avg }}$ of about $13-40 \%$. This can be done by scanning the fingerprint at the crime scene shortly after the crime investigation team arrives and within the next hours or even days (which is still practical, as our crime scene examiner contact told us) with the proposed surface measurement device using the CWL sensor. Having measured the relative amount of white background pixel $f_{E}\left(t_{E i}\right)$, we can easily calculate the age of the fingerprint by using formula (5). Since $e r r_{\text {avg }}$ only applies to an average case and $e r r_{i}$ can be much higher for a single scan, several scans should be carried out and the results should be averaged for a more reliable result.

However, the correct approximation of the parameters $a$ and $b$ remains a major challenge. To the best of our knowledge, it is not possible to obtain $a$ and $b$ directly from a crime scene without any additional information, regardless of how many scans are performed. This is due to the fact that neither the absolute time $t_{E i}$ of a scan is given, nor the parameter $a$ or the parameter $b$.

It is unlikely that $t_{E i}$ is given at a crime scene (since then the age determination would not be necessary). Therefore, either $a$ or $b$ need to be determined in advance. If one of the two parameters is known, the other one can be calculated by conducting only two scans of the fingerprint trace at the crime scene and solving the following system of equations: 


$$
\begin{gathered}
f_{M}\left(t_{M i}\right)=a * \ln \left(t_{M i}\right)+b, t_{M i}>0 . \\
f_{M}\left(t_{M i}+\Delta t\right)=a * \ln \left(t_{M i}+\Delta t\right)+b, t_{M i}>0, \Delta t>0 .
\end{gathered}
$$

Using this system of equations, $\Delta t$ represents the time between the two scans, $a$ or $b$ are given and $t_{M i}$ is to be substituted. If $a$ is given, $b$ can be calculated, if $b$ is given, $a$ can be calculated. Determining the parameter $b$ from a given parameter $a$ solving the system of equations (8)(9) and inserting the corresponding values $f_{E}\left(t_{E 2}\right)$ for $f_{M}\left(t_{M i}+\Delta t\right)$ and $f_{E}\left(t_{E l}\right)$ for $f_{M}\left(t_{M i}\right)$ whereas $\Delta t$ describes the time difference between the two scans $\Delta t=t_{E 2}-t_{E 1}$ results in the following formula:

$$
b=a * \ln \left(\frac{1}{\Delta t} *\left(e^{\frac{f_{E}\left(t_{E 2}\right)}{a}}-e^{\frac{f_{E}\left(t_{E 1}\right)}{a}}\right)\right), \Delta t>0, a \neq 0 .
$$

With the derived formula (10), the parameter $b$ can be calculated from a given parameter $a$ using at least two scans of the fingerprint trace. Again, in this situation it is recommended to use more than two scans for a better approximation of the average value of the parameters and minimising the influence of outliers.

The biggest problem remains the determination of one of the parameters $a$ or $b$ prior to the age determination. Since $b$ is strongly dependent on the amount of residue (as mentioned earlier), it is not possible to determine it in advance. However, it might be possible to determine the value of $a$ by taking all significant influences on the aging process into account. In future work, a generalised table might be created, which assigns certain values to $a$, according to the temperature, humidity, presence of UV-light or even chemical composition of the fingerprint residue.

\section{Conclusions and Future Work}

In this paper, the novel and very promising aging feature of counting binary pixel was examined in a test set of nine test series and a logarithmic course of its experimental aging curves was reported. A mathematical aging function was approximated for each test series as a continuous representation of the discrete experimental aging curve from the test results, to be used for the age determination of latent fingerprint traces in forensic investigations. The average error of approximation of this function was shown to be $13-40 \%$ under the given conditions. No characteristic influence of different resolutions or different sensors of the same model could be extracted within the test range. Possible reasons for the observed errors of approximation as well as prospects and limitations of an application of this function for the age determination of forensic latent fingerprint traces were discussed.

In future work, the average error of approximation of the function should further be decreased by taking additional measures to keep the influences on the aging process constant or to take changes of these influences into account. Here, additional studies need to be conducted to distinguish major influences from minor ones.

Furthermore, a technique should be investigated and developed for the reliable determination of the parameter $a$ of the proposed aging function prior to the age 
determination. This might be done by assigning certain values to the parameter depending on the specific characteristics of the present influences. Again, for this task, the significance of these influences needs to be studied further.

Acknowledgments. The work in this paper has been funded in part by the German Federal Ministry of Education and Science (BMBF) through the Research Programme under Contract No. FKZ: 13N10816 and FKZ: 13N10818. We also want to thank Anja Bräutigam from the Federal State Police Head Quarters of Saxony-Anhalt and Stefan Gruhn from the Brandenburg University of Applied Sciences for their support in conducting the scans.

\section{References}

1. Leich, M., Kiltz, S., Dittmann, J., Vielhauer, C.: Non-destructive forensic latent fingerprint acquisition with chromatic white light sensors. In: Proc. SPIE 7880, 78800S (2011), doi:10.1117/12.872331

2. Hildebrandt, M., Dittmann, J., Pocs, M., Ulrich, M., Merkel, R., Fries, T.: Privacy Preserving Challenges: New Design Aspects for Latent Fingerprint Detection Systems with Contact-Less Sensors for Future Preventive Applications in Airport Luggage Handling. In: Vielhauer, C., Dittmann, J., Drygajlo, A., Juul, N.C., Fairhurst, M.C. (eds.) BioID 2011. LNCS, vol. 6583, pp. 286-298. Springer, Heidelberg (2011)

3. Fries Research Technology (January 19, 2011), http: / /www. frt-gmbh. com/en/

4. Popa, G., Potorac, R., Preda, N.: Method for Fingerprints Age Determination (December 6, 2010), http: / / www . interpol . int/Public/Forensic/fingerprints / research/AgeDetermination.pdf

5. Stamm, V.: Methoden zur Altersbestimmung daktyloskopischer Spuren. Wiesbaden: 9. Grundlehrgang für daktyloskopische Sachverständige, Hausarbeit (1997)

6. Aehnlich, J.: Altersbestimmung von datkyloskopischen Spuren mit Hilfe der LaserFluoreszenzspektroskopie. Diplomarbeit, Universität Hannover (2001)

7. Baniuk, K.: Determination of Age of Fingerprints. Forensic Science International (46), 133-137 (1990)

8. Stüttgen, G., Ippen, H.: Die normale und pathologische Physiologie der Haut. G. Fischer Verlag, Stuttgart (1965)

9. Liappis, N., Jakel, A.: Free Amino Acids in Human Eccrine Sweat. Arch. Dermatol. Res. 254, 185-203 (1975)

10. Wertheim, K.: Fingerprint Age Determination: Is There Any Hope? Journal of Forensic Identification 53(1), 42-49 (2003)

11. Holyst, B.: Kriminalistische Abschätzung des Spurenalters bei Fingerpapillarlinien. Archiv für Kriminologie, 94-103 (1983)

12. Sampson, M.: Lifetime of a Latent Print on Glazed Ceramic Tile. Journal of Forensic Identification 44(4), 379-386 (1994) 